\title{
Hertz problem for a rigid punch moving across the surface of a semi-infinite elastic solid
}

\author{
M. Rahman
}

\begin{abstract}
The elastodynamic problem of a rigid punch moving at a constant sub-Rayleigh speed across the surface of an elastic half-space is investigated in the present paper. The unknown contact region is determined as part of solution from the unilateral or Signorini conditions. Numerical results are plotted showing how the eccentricity of the contact ellipse changes with the punch speed. Some asymptotic properties of the solution for the case where the punch speed is comparable with the Rayleigh wave speed are explored in details.
\end{abstract}

Mathematics Subject Classification (1991). 250H, 160B.

Keywords. Half-space, moving load, sub-Rayleigh speed, rigid punch, Hertz contact.

\section{Introduction}

A class of problems representing considerable physical interest in linear elastodynamics concerns the frictionless motion of a rigid punch at a velocity $V$ across the surface of an elastic half-space. In the case where the punch is a rigid cylinder or a rigid sphere of radius $R$, this problem is the elastodynamic counterpart of the classical Hertzian contact problems.

Churilov [1] treated the problem for the subsonic case $\left(V<c_{2}\right)$, where $c_{2}=$ $\sqrt{\mu / \rho}$ is the velocity of shear wave propagation ( $\mu$ being the shear modulus and $\rho$ the density of the material of the half-space). In an earlier paper [2], he derived an expression for the elastodynamic surface Green's function corresponding to the action of a normal load $P$, moving at some speed $V<c_{2}$, across the surface of an elastic half-space and then superposing this solution, he formulated the problem in the form of an integral equation in a frame of reference moving with the punch. He then developed a solution method but produced no numerical results. In the present article, the problem is analysed again. For the first time, numerical results are presented as to how the eccentricity of the contact ellipse changes with the punch speed. Some asymptotic properties of the solution concerning the case where the punch speed is comparable with the Rayleigh wave speed are explored at greater length. 


\section{The moving punch problem}

Obviously the problem under consideration has a steady-state solution in which the stress fields are invariant in a frame of reference moving with the punch and the contact area is a single connected region $\Omega$ in this frame of reference. Therefore we can reduce it to a classical elastodynamic boundary value problem.

If the boundary of the contact region $\partial \Omega$ is assumed to be given, the resulting boundary-value problem is well-posed. However the problem being physical demands that the contact area be determined as part of the solution from the unilateral or Signorini contact conditions which means that the contact tractions should be non-tensile throughout the contact region and the gap $g(x, y)$ between the half-space and the punch should be non-negative throughout the non-contact or separation region, i.e.

$$
\begin{aligned}
& p(x, y) \geq 0,(x, y) \in \Omega \\
& g(x, y) \geq 0,(x, y) \in\{z=0\} \backslash \Omega
\end{aligned}
$$

It can be shown (see for example [3]) that these inequalities are equivalent to the conditions requiring that the contact tractions be non-tensile throughout the contact region and that it vanish at the edges of the contact region, i.e.

$$
\begin{aligned}
& p(x, y) \geq 0, \quad(x, y) \in \Omega \\
& p(x, y)=0,(x, y) \in \partial \Omega
\end{aligned}
$$

Characteristic of this kind of problems where the contact region is not given $a$ priori is the fact that the contact region changes with the indenting force $P$. In general such problems are non-linear and therefore closed-form solutions of such problems can be derived only in exclusive cases. Iterative or numerical schemes are usually employed to solve this kind of problems. However we should note that the boundary conditions (2) and (3) are very sensitive to iterative or numerical treatment of such problems, since the assignment of an initial contact region which is bigger than the actual one leads to the violation of the condition (2), whilst the converse leads to the violation of the condition (3).

\section{The elastodynamic surface Green's function}

The problem under consideration can be efficiently formulated in the form of an integral equation involving the appropriate Green's function, which corresponds to the response of the elastic half-space to a point normal load $P$ moving along the $x$-axis at a speed $V$ across the surface $z=0$. This problem was attacked by many authors. A convenient summary of the work in this direction can be found in Eringen and Suhubi [4]. It was first analysed by Payton [5] using the BettiRayleigh theorem. However his results are restricted to Poisson's materials only. 
Eason [6] employed integral transform technique to solve this problem, but was able to find the surface Green's function for the sub-Rayleigh case only. Churilov [1] extended this result for the case of sub-shear speed, using a technique due to Smirnov and Sobolev [7]. The expression he derived for the surface displacement in a frame of reference moving with the load $P$ is as follows:

$$
u_{z}(x, y, 0)=-\frac{P}{2 \mu r} \Gamma\left(\frac{V y}{r}\right), 0<V<c_{2}
$$

where $r=\sqrt{x^{2}+y^{2}}$ and the function $\Gamma(V)$ is given by the formula

$$
\Gamma(V)=\frac{M_{2}^{2} \sqrt{1-M_{1}^{2}}}{\pi R(V)}, 0<V<c_{2}
$$

where

$$
R(V)=\left(2-M_{2}^{2}\right)^{2}-4 \sqrt{\left(1-M_{1}^{2}\right)\left(1-M_{2}^{2}\right)}
$$

is the Rayleigh function and $M_{1}=V / c_{1}$ and $M_{2}=V / c_{2}$ are the longitudinal and shear wave Mach numbers, respectively.

The problem of derivation of the elastodynamic surface Green's functions for the transonic and supersonic cases is still an open question and is currently under the writer's investigation.

\section{Integral equation of the problem and derivation of the Hertzian equations}

The elastodynamic surface Green's function (4) allows an elegant formulation of the title problem. We can write down the normal surface displacement in a region $\Omega$ due to an arbitrary contact pressure $p(x, y)$, in the form of a convolution integral, i.e.

$$
u_{z}(x, y, 0)=-\frac{1}{2 \mu} \int_{\Omega} \int \frac{p\left(x_{1}, y_{1}\right)}{R} \Gamma\left\{\frac{V\left(y-y_{1}\right)}{R}\right\} d x_{1} d y_{1}
$$

where $R=\sqrt{\left(x-x_{1}\right)^{2}+\left(y-y_{1}\right)^{2}}$ is the distance between the points $P(x, y)$ and $P^{\prime}\left(x_{1}, y_{1}\right)$ (Fig.1).

The corresponding integral equation for the static case can be derived by letting $V$ tend to zero in (5), (6) and (7), namely,

$$
u_{z}(x, y, 0)=\frac{1-\nu}{2 \pi \mu} \int_{\Omega} \int \frac{p\left(x_{1}, y_{1}\right)}{R} d x_{1} d y_{1}
$$

A general solution of the integral equation of the type (8) for the case where $\Omega$ is an elliptical region is given by Galin [8] who shows that if the function $u_{z}(x, y, 0)$ 


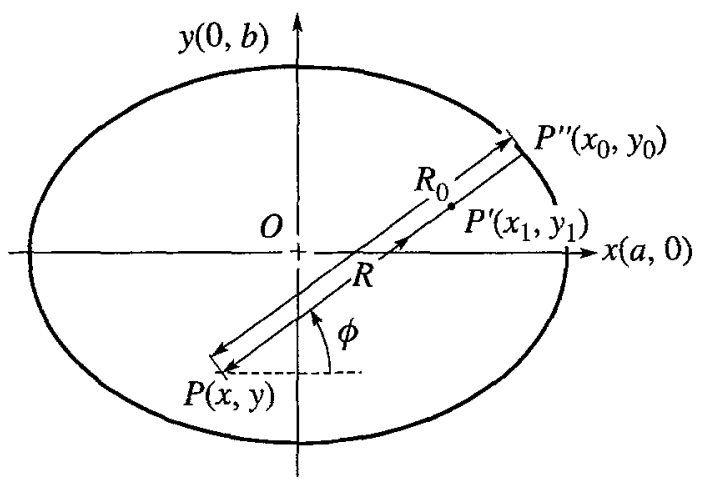

Figure 1.

Elliptical Contact Region

is a polynomial of $x$ and $y$ of order $2 m$, the solution for the function $p(x, y)$ has the form:

$$
p(x, y)=p_{0}\left(1-\frac{x^{2}}{a^{2}}-\frac{y^{2}}{b^{2}}\right)^{m-\frac{1}{2}}
$$

where $p_{0}$ is an unknown coefficient. In literature this is known as Galin's theorem, although as pointed out by Cherepanov [9], a theorem much stronger than Galin's was proved by Dyson in 1891 [10]. In what follows we will show that Galin's theorem can still be employed to solve the elastodynamic equation (7). In so doing, we will closely follow Vorovich et al [11].

From the geometry of the Figure 1, we have

$$
\begin{aligned}
& x_{1}=x+R \cos \phi \\
& y_{1}=y+R \sin \phi
\end{aligned}
$$

Substituting (10) into (9), we obtain

$$
\tilde{p}(R, \phi)=p(x+R \cos \phi, y+R \sin \phi)=p_{0}\left\{\left(A-2 B(\phi) R-C(\phi) R^{2}\right)\right\}^{m-\frac{1}{2}}
$$

where

$$
\begin{aligned}
& A=1-\frac{x^{2}}{a^{2}}-\frac{y^{2}}{b^{2}} \\
& B=\frac{x \cos \phi}{a^{2}}+\frac{y \sin \theta}{b^{2}} \\
& C=\frac{\cos ^{2} \phi}{a^{2}}+\frac{\sin ^{2} \phi}{b^{2}}
\end{aligned}
$$

Note that the functions $B(\phi)$ and $C(\phi)$ have the following properties:

$$
B(\pi+\phi)=-B(\phi), C(\pi+\phi)=C(\phi)
$$


Now putting (10) and (11) into (7), and considering that $\Gamma(V)$ is an even function of $V$, we get

$$
\begin{aligned}
u_{z}(x, y, 0) & =\frac{1}{2 \mu} \int_{0}^{2 \pi} \int_{0}^{R_{0}(\phi)} \tilde{p}(R, \phi) \Gamma(V \sin \phi) d R d \phi \\
& =-\frac{p_{0}}{2 \mu} \int_{0}^{2 \pi} \int_{0}^{R_{0}(\phi)}\left\{A-2 B(\phi) R-C(\phi) R^{2}\right\}^{m-\frac{1}{2}} \Gamma(V \sin \phi) d R d \phi
\end{aligned}
$$

where $R_{0}(\phi)$ is the distance between the points $P$ and $P^{\prime \prime}$ (Fig. 1).

Note that since the point $P^{\prime \prime}$ lies on the boundary of the ellipse, the following equations hold:

$$
\begin{aligned}
& \frac{x_{0}^{2}}{a^{2}}+\frac{y_{0}^{2}}{b^{2}}=1 \\
& x_{0}=x+R_{0} \cos \phi \\
& y_{0}=y+R_{0} \sin \phi
\end{aligned}
$$

Using equations (15) and the notations (12), it can be shown that

$$
R_{0}(\phi)=\frac{-B(\phi)+\sqrt{B^{2}(\phi)+A C(\phi)}}{C(\phi)}
$$

Since the point $P(x, y)$ lies within the ellipse (Fig. 1), $A(\phi)>0$, and $C(\phi)>0$ for all $\phi \in[0,2 \pi]$. Therefore

$$
\frac{B}{\sqrt{B^{2}+A C}} \leq \frac{B+R C}{\sqrt{B^{2}+A C}} \leq \frac{B+R_{0} C}{\sqrt{B^{2}+A C}}
$$

Besides, it can be easily shown that $B /\left(B^{2}+A C\right)>-1$ and $\left(B+R_{0} C\right) /\left(\sqrt{B^{2}+A C}\right)=$ 1 (by virtue of (16)). Therefore the following relationship holds:

$$
-1<\frac{B+R C}{\sqrt{B^{2}+A C}} \leq 1
$$

Now introducing a new variable $\theta$ by the relation

$$
\cos \theta=\frac{B+R C}{\sqrt{B^{2}+A C}}, \quad(0 \leq \theta \leq \pi)
$$

equation (14) can be rewritten as below

$u_{z}(x, y, 0)=-\frac{p_{0}}{2 \mu} \int_{0}^{2 \pi} \int_{0}^{\theta(\phi)} \frac{1}{\sqrt{C(\phi)}}\left(\frac{B^{2}(\phi)+A C(\phi)}{C(\phi)}\right)^{m} \sin ^{2 m} \theta \Gamma(V \sin \phi) d \theta d \phi$ 
where $\theta(\phi)$ is the value of $\theta$ corresponding to $R=0$.

At this stage, consider the integral

$$
I=\int_{\pi}^{2 \pi} \int_{0}^{\theta(\phi)} \frac{1}{\sqrt{C(\phi)}}\left(\frac{B^{2}(\phi)+A C(\phi)}{C(\phi)}\right)^{m} \sin ^{2 m} \theta \Gamma(V \sin \phi) d \theta d \phi
$$

We now put $\phi=\pi+\psi$ into (21) and note that $\cos \theta(\pi+\psi)=-\cos \theta(\psi)$ whence it follows that $\theta(\pi+\psi)=\pi-\theta(\psi)$. In view of this and the properties of the functions $B(\phi)$ and $C(\phi)$ defined by the equations (13), from (21) we get

$$
I=\int_{0}^{\pi} \int_{0}^{\pi-\theta(\psi)} \frac{1}{\sqrt{C(\psi)}}\left(\frac{B^{2}(\psi)+A C(\psi)}{C(\psi)}\right)^{m} \sin ^{2 m} \theta \Gamma(-V \sin \psi) d \theta d \psi
$$

Now putting $\theta=\pi-\theta^{\prime}$ into (22) and again noting that $\Gamma(V)$ is an even function of $V$, we obtain

$$
I=\int_{0}^{\pi} d \psi \int_{\theta(\psi)}^{\pi} \frac{1}{\sqrt{C(\psi)}}\left(\frac{B^{2}(\psi)+A C(\psi)}{C(\psi)}\right)^{m} \sin ^{2 m} \theta^{\prime} \Gamma(V \sin \psi) d \theta^{\prime} d \psi
$$

With the help of equation (23), we represent equation (20) as

$$
u_{z}(x, y, 0)=-\frac{p_{0}}{2 \mu} \int_{0}^{\pi} \int_{0}^{\pi} \frac{1}{\sqrt{C(\phi)}}\left(\frac{B^{2}(\phi)+A C(\phi)}{C(\phi)}\right)^{m} \sin ^{2 m} \theta \Gamma(V \sin \phi) d \theta d \phi
$$

The integral with respect to $\theta$ in (24) can be evaluated in closed form yielding the following expression for $u_{z}(x, y, 0)$ :

$$
u_{z}(x, y, 0)=-\frac{p_{0}(2 m-1) ! ! \pi}{2 \mu(2 m) ! !} \int_{0}^{\pi} \frac{1}{\sqrt{C(\phi)}}\left(\frac{B^{2}(\phi)+A C(\phi)}{C(\phi)}\right)^{m} \Gamma(V \sin \phi) d \phi
$$

Since $C(\phi)$ and $\Gamma(V \sin \phi)$ are independent of $x$ and $y$, from (25), we conclude that $u_{z}(x, y, 0)$ is a polynomial in $x$ and $y$ of degree $2 m$.

We now turn to the Hertz problem.

\section{Hertz problem}

For the special case where $m=1$, we have

$$
u_{z}(x, y, 0)=-\frac{p_{0} \pi}{4 \mu} \int_{0}^{\pi} \frac{B^{2}(\phi)+A C(\phi)}{C^{3 / 2}(\phi)} \Gamma(V \sin \phi) d \phi
$$

which can be written in the following more convenient form:

$$
u_{z}(x, y, 0)=d_{0}-A_{0} x^{2}-B_{0} y^{2}
$$


where

$$
\begin{aligned}
& d_{0}=\frac{p_{0} b}{4 \mu} \int_{0}^{\pi} \frac{\Gamma(V \sin \phi)}{\left(k^{2} \cos ^{2} \phi+\sin ^{2} \phi\right)^{\frac{1}{2}}} d \phi \\
& A_{0}=-\frac{p_{0} k}{4 \mu a} \int_{0}^{\pi} \frac{\Gamma(V \sin \phi) \sin ^{2} \phi}{\left(k^{2} \cos ^{2} \phi+\sin ^{2} \phi\right)^{\frac{3}{2}}} d \phi \\
& B_{0}=-\frac{p_{0} k}{4 \mu a} \int_{0}^{\pi} \frac{\Gamma(V \sin \phi) \cos ^{2} \phi}{\left(k^{2} \cos ^{2} \phi+\sin ^{2} \phi\right)^{\frac{3}{2}}} d \phi
\end{aligned}
$$

where $k=b / a$.

In deriving the above equation, we have made use of the fact that the integral with the $x y$ term is zero, since the integrand is an odd function.

Now suppose the elastic half-space is indented by a rigid indenter whose profile is defined by the gap function

$$
g(x, y)=A_{1} x^{2}+B_{1} y^{2}=\frac{x^{2}}{2 R_{1}}+\frac{y^{2}}{2 R_{2}}
$$

where $R_{1}$ and $R_{2}$ are the principal radii of curvature. It is assumed that $R_{1}>R_{2}$ or $A_{1}<B_{1}$. We equate the coefficients between the equations (27) and (29) to obtain two equations for the unknown semi-axes $a, b$ of the contact ellipse and the central pressure $p_{0}$, which are

$$
\begin{aligned}
& A_{1}=\frac{1}{2 R_{1}}=-\frac{p_{0} k}{4 \mu a} \int_{0}^{\pi} \frac{\Gamma(V \sin \phi) \sin ^{2} \phi}{\left(k^{2} \cos ^{2} \phi+\sin ^{2} \phi\right)^{\frac{3}{2}}} d \phi \\
& B_{1}=\frac{1}{2 R_{2}}=-\frac{p_{0} k}{4 \mu a} \int_{0}^{\pi} \frac{\Gamma(V \sin \phi) \cos ^{2} \phi}{\left(k^{2} \cos ^{2} \phi+\sin ^{2} \phi\right)^{\frac{3}{2}}} d \phi
\end{aligned}
$$

To obtain a third equation, we note that either the total force $P$ or the central displacement $d_{0}$ must be given. If $P$ is given, we note that

$$
P=4 \int_{0}^{a} \int_{0}^{b \sqrt{1-x^{2}}} p_{0}\left(1-\frac{x^{2}}{a^{2}}-\frac{y^{2}}{b^{2}}\right)^{\frac{1}{2}} d y d x=\frac{2}{3} \pi p_{0} a b
$$

Alternatively, if $d_{0}$ is given, we have

$$
d_{0}=\frac{p_{0} b}{4 \pi \mu} \int_{0}^{\pi} \frac{\Gamma(V \sin \phi)}{\left(k^{2} \cos ^{2} \phi+\sin ^{2} \phi\right)^{\frac{1}{2}}} d \phi
$$

The resulting simultaneous equations are non-linear in $a, b$. An iterative, numerical or graphical method must be used. However, we note that a single equation depending on the ellipticity, $k=b / a$ or the eccentricity, $e=\sqrt{1-k^{2}}$, of the contact ellipse and $V$ can be obtained from the ratio of the two equations (30), i.e.

$$
\frac{A_{1}}{B_{1}}=\frac{R_{2}}{R_{1}}=\frac{I_{2}}{I_{1}}
$$


where

$$
\begin{aligned}
& I_{1}=-\int_{0}^{\pi} \frac{\Gamma(V \sin \phi) \cos ^{2} \phi}{\left(k^{2} \cos ^{2} \phi+\sin ^{2} \phi\right)^{\frac{3}{2}}} d \phi \\
& I_{2}=-\int_{0}^{\pi} \frac{\Gamma(V \sin \phi) \sin ^{2} \phi}{\left(k^{2} \cos ^{2} \phi+\sin ^{2} \phi\right)^{\frac{3}{2}}} d \phi
\end{aligned}
$$

Thus, the eccentricity $e$ of the contact ellipse depends on the ratio $R_{2} / R_{1}$ or $A_{1} / B_{1}$, Poisson's ratio $\nu$ and the punch speed $V$ in a very complicated way.

\section{Series solution of the problem}

The question as to how the ellipticity of the contact ellipse changes with the speed of the indenter necessitates evaluation of the integrals (34). In all probability, they can not be evaluated in closed forms, but they are amenable to effective numerical evaluation. However a series solution of the problem can be derived by representing the function $\Gamma(V \sin \theta)$ in the form of a power series of $\sin ^{2} \theta$. This is based on rationalizing the fraction in the expression of $\Gamma(V \sin \theta)$ and then expanding the radicals in binomial series. The final result is:

$$
\Gamma(V \sin \phi)=\sum_{n=0}^{\infty} f_{n} \sin ^{2 n} \phi
$$

The coefficients $f_{n}$ are determined from the following recurrence relationships:

$$
\begin{aligned}
A f_{l}+B f_{l-1} & +C f_{l-2}+D f_{l-3}=-M_{1}^{2 l-4} M_{2}^{6} \frac{(2 l-7) ! !}{(l-2) ! 2^{l-2}}+4 M_{1}^{2 l-2} M_{2}^{4} \frac{(2 l-5) ! !}{(l-1) ! 2^{l-1}} \\
& +4 M_{1}^{2} M_{2}^{2 l} \frac{(2 l-5) ! !}{(l-1) ! 2^{l-1}}-4 M_{1}^{2 l} M_{2}^{2} \frac{(2 l-3) ! !}{l ! 2^{l}}-4 M_{2}^{2 l+2} \frac{(2 l-3) ! !}{l ! 2^{l}}(l>2)
\end{aligned}
$$

where

$$
\begin{array}{r}
f_{0}=1-\nu \\
f_{1}=\frac{M_{2}^{2}}{16}\left(16 \nu^{2}-24 \nu+14\right) \\
f_{2}=\frac{M_{2}^{4}}{64(1-\nu)}\left(27-220 \nu+312 \nu^{2}-224 \nu^{3}+64 \nu^{4}\right) \\
A=8 \frac{M_{2}^{2}}{1-\nu} \\
B=-\frac{8(2-\nu) M_{2}^{4}}{1-\nu} \\
C=8 M_{2}^{6} \\
D=-M_{2}^{8}
\end{array}
$$


In expression (36), it is assumed that $(-1) ! !=1$.

It can be shown that the series (36) is uniformly convergent for the case where the punch speed is less than the Rayleigh wave speed.

Now putting the expansion (36) into (34), we obtain

$$
\begin{aligned}
& I_{1}=2 \sum_{n=0}^{\infty} f_{n}\left(L_{n}-L_{n+1}\right) \\
& I_{2}=2 \sum_{n=0}^{\infty} f_{n} L_{n+1}
\end{aligned}
$$

where the following notation is introduced:

$$
L_{n}=\frac{1}{2} \int_{0}^{\pi} \frac{\sin ^{2 n} \phi}{\left(k^{2} \cos ^{2} \phi+\sin ^{2} \phi\right)^{\frac{3}{2}}} d \phi
$$

By making a change of variable $\phi=\pi / 2-\alpha$ in (38), and in view of the symmetry of the resulting integral, it can be rewritten as:

$$
L_{n}=\int_{0}^{\frac{\pi}{2}} \frac{\cos ^{2 n} \alpha d \alpha}{\left(1-e^{2} \sin ^{2} \alpha\right)^{\frac{3}{2}}}
$$

Closed-form expressions can be obtained for the integral (39) for all values of $n$, using the following recursion relations derived in Appendix A:

$$
\begin{aligned}
L_{n}= & \frac{4(n-1)}{(2 n-1) e^{2}} M_{n-1}+\frac{2(n-1)\left(2 e^{2}-1\right)}{(2 n-1) e^{2}} L_{n-1}-\frac{2(2 n-3)}{(2 n-1) e^{2}} M_{n-2} \\
& +\frac{(2 n-3)\left(1-e^{2}\right)}{(2 n-1) e^{2}} L_{n-2} \\
& M_{n}=\frac{2(n-1)\left(2 e^{2}-1\right)}{(2 n-1) e^{2}} M_{n-1}+\frac{(2 n-3)\left(1-e^{2}\right)}{(2 n-1) e^{2}} M_{n-2}
\end{aligned}
$$

where $M_{n}$ stands for

$$
M_{n}=\int_{0}^{\frac{\pi}{2}} \frac{\cos ^{2 n} \alpha d \alpha}{\left(1-e^{2} \sin ^{2} \alpha\right)^{\frac{1}{2}}}
$$

The recursion expressions (40) are valid for $n \geq 2$. Below are given the starting values of $L_{n}$ and $M_{n}$, namely for $n=0$ and $n=1$,

$$
\begin{aligned}
L_{0} & =\frac{1}{k^{2}} E(e) \\
L_{1} & =\frac{1}{e^{2}}[K(e)-E(e)] \\
M_{0} & =K(e) \\
M_{1} & =\frac{1}{e^{2}}\left[E(e)-k^{2} K(e)\right]
\end{aligned}
$$


where $E(e)$ and $K(e)$ are the complete elliptical integrals of the first and second kinds, respectively.

In the limiting case where $V \rightarrow 0$, all the coefficients $f_{n}$ except $f_{0}$ reduce to zero and we get corresponding static solution

$$
\begin{aligned}
& I_{1}=\frac{2(1-\nu)}{e^{2}}\left[\frac{E(e)}{k^{2}}-K(e)\right] \\
& I_{2}=\frac{2(1-\nu)}{e^{2}}[K(e)-E(e)]
\end{aligned}
$$

which is precisely the same as that given in [3] \& [12].

\section{Modification of the expressions for $I_{1}(V)$ and $I_{2}(V)$}

The foregoing analysis is best suited for comparatively small values of the Rayleigh wave number $M_{R}$. If the speed of the indenter is comparable with the Rayleigh wave speed, then at $\phi=\pi / 2$ the numerator of the integrands in (34) reduces to the Rayleigh function which, as it is well-known, has a simple zero at $M_{R}=1$. The series solution of the problem represented by the equations (37) will be converging very slowly. For this case, we proceed with using the factorized form of the function $R(V)$ (eqn (6)) [13]

$$
R(V)=\frac{M_{2}^{2}\left(M_{2}^{2}-m_{1}\right)\left(M_{2}^{2}-m_{2}\right)\left(M_{2}^{2}-m_{3}\right)}{\left(2-M_{2}^{2}\right)^{4}+4 \sqrt{\left(1-M_{1}^{2}\right)\left(1-M_{2}^{2}\right)}}
$$

where $m_{1}, m_{2}$ and $m_{3}$ are the three roots of the Rayleigh function, $m_{1}$ being the Rayleigh root. Explicit expressions for these roots are given in [13].

In view of the above factorized form of $R(V)$, we now represent the function $\Gamma(V)$ (see eqn (5)) as below:

$$
\Gamma(V)=-\frac{G(V)}{1-M_{R}^{2}}
$$

where $G(V)$ is given by

$$
G(V)=\frac{\sqrt{1-M_{1}^{2}}\left\{\left(2-M_{2}^{2}\right)^{2}+4 \sqrt{\left(1-M_{1}^{2}\right)\left(1-M_{2}^{2}\right)}\right\}}{\pi m_{1}\left(M_{2}^{2}-m_{2}\right)\left(M_{2}^{2}-m_{3}\right)}
$$

It can be shown that $G(V)$ is a monotone decreasing function of $V$ for all $V \in$ $\left[0, c_{R}\right]$.

Putting (44) into (34) and making a change of variable $\phi=\pi / 2-\alpha$, we obtain

$$
\begin{aligned}
& I_{1}=2 \int_{0}^{\pi / 2} \frac{G(V \cos \alpha) \sin ^{2} \alpha d \alpha}{\left(1-M_{R}^{2} \cos ^{2} \alpha\right) \triangle^{3}} \\
& I_{2}=2 \int_{0}^{\pi / 2} \frac{G(V \cos \alpha) \cos ^{2} \alpha d \alpha}{\left(1-M_{R}^{2} \cos ^{2} \alpha\right) \triangle^{3}}
\end{aligned}
$$


where $\triangle=\sqrt{1-e^{2} \sin ^{2} \alpha}$.

Now it is easy to see from (46) and (47) that for fixed $k$, integral (46) is bounded for all $M_{R} \in[0,1]$, while the integral (47) becomes unbounded at $\alpha=0$ and $M_{R} \rightarrow 1 \ldots$. We therefore represent the integral (47) as

$$
I_{2}=2 G(V) S+2 \int_{0}^{\pi / 2} \frac{G(V \cos \alpha)-G(V)}{\left(1-M_{R}^{2} \cos ^{2} \alpha\right) \triangle^{3}} \cos ^{2} \alpha d \alpha
$$

where

$$
S=\int_{0}^{\pi / 2} \frac{\cos ^{2} \alpha d \alpha}{\left(1-M_{R}^{2} \cos ^{2} \alpha\right) \triangle^{3}}
$$

Now for fixed $k$, the second integral in (48) is bounded for all $M_{R} \in\left[0, c_{R}\right]$. Therefore the singular behavior of the integral $I_{2}$ at $M_{R} \rightarrow 1_{-}$is determined solely by the integral $S$ given by (49). Therefore in what follows we will explore the asymptotic behavior of this particular integral for $M_{R} \rightarrow 1_{-}$.

Note that $S$ can be represented as below:

$$
S=e S_{1}^{\prime}(e)+S_{1}(e)
$$

where

$$
S_{1}=\int_{0}^{\pi / 2} \frac{\cos ^{2} \alpha d \alpha}{\left(1-M_{R}^{2} \cos ^{2} \alpha\right) \triangle}
$$

Integral (51) is evaluated in closed form using the standard integral 3.679.1 from Gradshteyn and Ryzhik [14]:

$$
\begin{aligned}
S_{1}=\frac{1}{M_{R} \sqrt{1-M_{R}^{2}} \sqrt{1-k^{2}\left(1-M_{R}^{2}\right)}} & {\left[\frac{\pi}{2}-K(e) E(\beta, k)-E(e) F(\beta, k)\right.} \\
& +K(e) F(\beta, k)]
\end{aligned}
$$

where $\beta=\arccos M_{R}$, and $E(\beta, k)$ and $F(\beta, k)$ are the incomplete elliptical integrals of the first and second kinds, respectively, $E(e)=E(\pi / 2, e)$ and $K(e)=$ $F(\pi / 2, e)$ are the complete elliptical integrals of the first and second kinds, respectively.

Insertion of (52) into (50) leads to the following expression for $S$ :

$$
\begin{aligned}
S= & \frac{1}{1-k^{2}\left(1-M_{R}^{2}\right)}[K(e)-E(e)]+\frac{M_{R}}{\sqrt{1-M_{R}^{2}\left\{1-k^{2}\left(1-M_{R}^{2}\right)\right\}^{3 / 2}}} \\
& {\left[\frac{\pi}{2}-K(e) E(\beta, k)-E(e) F(\beta, k)+K(e) F(\beta, k)\right] }
\end{aligned}
$$

In deriving (53), use has been made of the following differentiation rule for the elliptical integrals [14]:

$$
\begin{aligned}
& \frac{\partial E(\beta, p)}{\partial p}=\frac{1}{p}[E(\beta, p)-F(\beta, p)] \\
& \frac{\partial F(\beta, p)}{\partial p}=\frac{1}{1-p^{2}}\left[\frac{1}{p} E(\beta, p)-\frac{1-p^{2}}{p} F(\beta, p)-\frac{p \sin 2 \beta}{2 \sqrt{1-p^{2} \sin ^{2} \beta}}\right]
\end{aligned}
$$




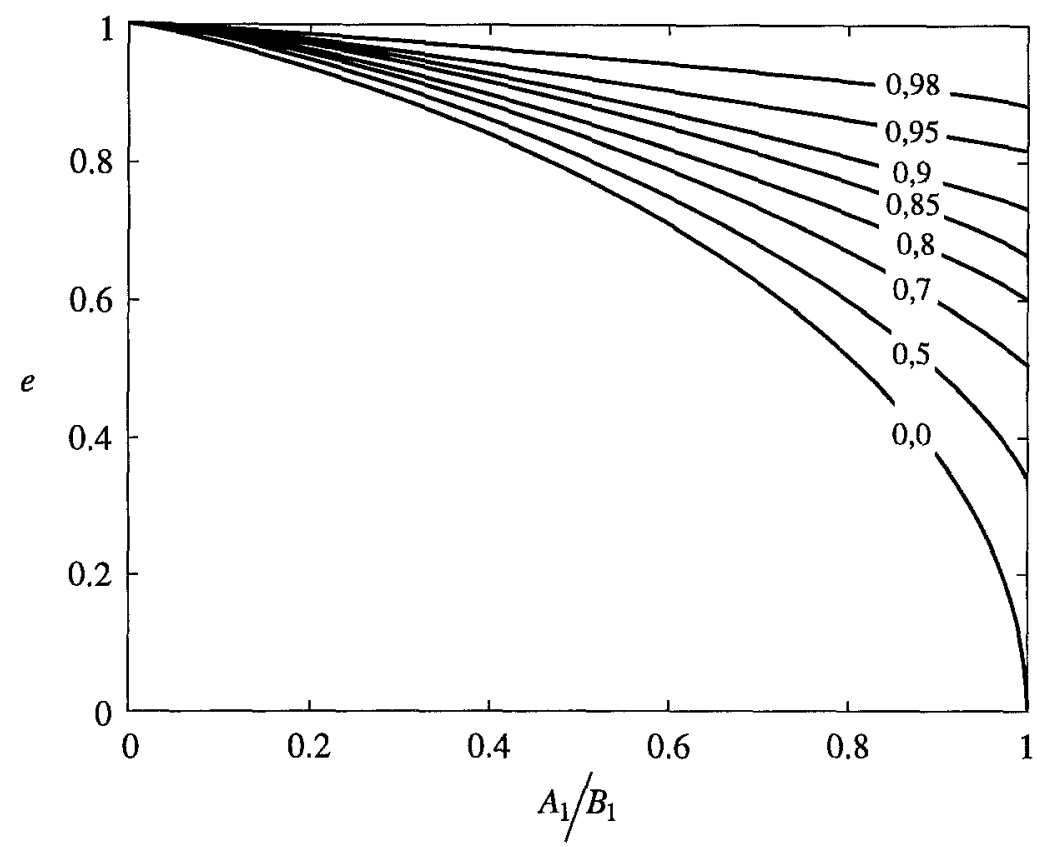

Figure 2.

Variations of the eccentricity of the contact ellipse with the ratio $A_{1} / B_{1}$ for different values of the Rayleigh wave number

From equations (48) and (53), we observe that the integral $I_{2}(V)$ behaves as $C\left(1-M_{R}\right)^{-1 / 2}\left(C\right.$ being a finite constant) as $M_{R} \rightarrow 1$.

\section{Numerical results and discussion}

The problem has also been analysed by evaluating the integrals (34) numerically. While $I_{1}$ has been evaluated as per $\left(34_{1}\right), I_{2}$ has been evaluated by using the expressions (48) and (53) instead of the expression $\left(34_{2}\right)$. Numerical results have been obtained for a wide range of values for $M_{R}$ and Poisson's ratio, $\nu$. Some of the results thus obtained are represented in Fig. 2. It has been observed that the effect of Poisson's ratio on the numerical results is of the order of $2 \%$. Therefore graphical representation of the numerical results is done only for the case, $\nu=0.2$. For small values of the Rayleigh wave number, our numerical results are found to be in excellent agreement with the corresponding static results $[3,12]$. 


\section{Concluding remarks}

In the present paper, we have investigated the elastodynamic Hertz problem for a rigid indenter moving over the surface of an elastic half-space. For the first time numerical results have been presented as to how the ellipticity of the contact ellipse changes with the speed of the indenter. Some asymptotic estimates of the solution for the case where the speed of the indenter is comparable with the Rayleigh wave speed have been explored in details. Of further interest is the corresponding problem for a slender indenter for which the present solution is not much suitable. For this case, it seems promising to use the method of matched asymptotic expansions in conjunction with the present analysis. Research in this direction will be reported elsewhere.

\section{Acknowledgment}

The research reported in this article was carried out while the writer was a Visiting Scholar at the College of Engineering, University of Michigan, Ann Arbor, USA. In this connection, he would like to acknowledge with gratitude supervision and partial financial support of Professor J. R. Barber.

\section{References}

[1] V. A. Churilov, On the effect of a normal load moving at a constant velocity along the boundary of an elastic half-space. J. Appl. Math. Mech., 41 (1977), 125-132.

[2] V. A. Churilov, Action of an elliptical stamp moving at a constant speed on an elastic half-space. J. Appl. Math. Mech. 42 (1978). 1176-1182.

[3] L. A. Galin, Contact problems of the theory of elasticity and viscoelasticity. Moscow, Nauka, 1980. (In Russian)

[4] A. C. Eringen and E. S. Suhubu, Elastodynamics. Vol. II: Linear Theory. Academic Press, New York, 1975.

[5] R. G. Payton, An application of the dynamic Betti-Rayleigh reciprocal theorem to moving point loads in elastic media. Quart. Appl. Math. 21 (1964), 299-313.

[6] G. Eason, The stresses produced in a semi-infinite solid by a moving surface load. Int. $J$. Engg. Sci., 2(6) (1965), 581-609.

[7] V. I. Smirnov and S. G. Sobolev, Sur le problème plan des vibrations élastiques. C. $R$. Acad. Sci. Paris, 194 (1932), 1437-1439.

[8] L. A. Galin, On the pressure of a stamp of elliptical planform on an elastic half-space. Prikladnaya Matematika i Mekhanika(PMM), 11, 2 (1947) (in Russian).

[9] G. P. Cherepanov, Mechanics of Brittle Fracture. McGraw-Hill, New York, 1979.

[10] F. W. Dyson, The potentials of ellipsoids of variable densities. Quart. J. Math., Oxford Ser., XXV (1891) 259-288.

[11] I. I. Vorovich, V. M. Alexandrov and V. A. Babeshko, Non-classical Mixed Boundary Value Problems of the Theory of Elasticity. Nauka, Moscow, 1974 (in Russian).

[12] I. I. Shtaerman, Contact Problems of the Theory of Elasticity. ONTI, Moscow-Leningrad, 1949 (In Russian).

[13] M. Rahman and J. R. Barber, Exact expressions for the roots of the secular equation for 
Rayleigh waves. ASME Journal of Applied Mechanics 62(1) (1995), 250-252.

[14] I. S. Gradshteyn and I. M. Ryzhik, Tables of Integrals, Series and Products. Academic Press, New York, 1980.

\section{Appendix}

The objective of this appendix is to develop a recursion relation for $L_{n}$ (see eqn (38)).

From [14, p. 158], we have a recursion formula for the following integral:

$$
M_{n}=\int_{0}^{\pi / 2} \frac{\cos ^{2 n} \alpha d \alpha}{\left(1-e^{2} \sin ^{2} \alpha\right)^{1 / 2}}
$$

as

$$
M_{n}=\frac{(2 n-2)\left(2 e^{2}-1\right)}{(2 n-1) e^{2}} M_{n-1}+\frac{(2 n-3)\left(1-e^{2}\right)}{(2 n-1) e^{2}} M_{n-2}
$$

We now represent the integral (A.1) as below:

$$
M_{n}=p \int_{0}^{\pi / 2} \frac{\cos ^{2 n} \alpha d \alpha}{\left(p^{2}-\sin ^{2} \alpha\right)^{1 / 2}}
$$

where $p=1 / e$.

Differentiating both sides of (A.3) with respect to $p$, we get

$$
\frac{d M_{n}}{d p}=-p^{2} \int_{0}^{\pi / 2} \frac{\cos ^{2 n} \alpha d \alpha}{\left(p^{2}-\sin ^{2} \alpha\right)^{3 / 2}}+\int_{0}^{\pi / 2} \frac{\cos ^{2 n} \alpha d \alpha}{\left(p^{2}-\sin ^{2} \alpha\right)^{1 / 2}}
$$

Again putting $p=1 / e$ into the right hand side of (A.4) and using the notations $L_{n}$ and $M_{n}$, we obtain

$$
\frac{d M_{n}}{d p}=-e L_{n}+e M_{n}
$$

We now differentiate the expression (A.2) with respect to $p$, obtaining

$$
\frac{d M_{n}}{d p}=\frac{2(1-p)\left(2-p^{2}\right)}{2-p} \frac{d M_{n-1}}{d p}+\frac{(2-3 p)\left(p^{2}-1\right)}{(2-p)} \frac{d M_{n-2}}{d p}-\frac{4 p(1-p)}{(2-p)} M_{n-1}+\frac{2 p(2-3 p)}{(2-p)} M_{n-2}
$$

For the derivatives of $d M_{n}(p) / d p, d M_{n-1} / d p$ and $d M_{n-2} / d p$, we use the relation (A.5) and performing some routine manipulations, we finally get the following recursion formula for $L_{n}$ :

$$
L_{n}=\frac{4(n-1)}{(2 n-1) e^{2}} M_{n-1}+\frac{2(n-1)\left(2 e^{2}-1\right)}{(2 n-1) e^{2}} L_{n-1}-\frac{2(2 n-3)}{(2 n-1) e^{2}} M_{n-2}+\frac{(2 n-3)\left(1-e^{2}\right)}{(2 n-1) e^{2}} L_{n-2}
$$

where $M_{n}$ is expressed through the recursion formula (A.2).

The recursion expressions (A.2) and (A.7) are valid for $n \geq 2$. The starting values for (A.2) and (A.7) are

$$
\begin{aligned}
L_{0} & =\frac{1}{k^{2}} E(e) \\
L_{1} & =\frac{1}{e^{2}}[K(e)-E(e)] \\
M_{0} & =K(e) \\
M_{1} & =\frac{1}{e^{2}}\left[E(e)-k^{2} K(e)\right]
\end{aligned}
$$


where $E(e)$ and $K(e)$ are the complete elliptical integrals of the first and second kinds, respectively. Expressions (A.8) are obtained by using the standard integrals 2.584.1, 2.584.6, 2.584.42 and 2.584.51 from [14].

M. Rahman

Department of Aerospace Engineering

University of Michigan, Ann Arbor, MI 48109, USA

Address for correspondence:

2715 Windwood Dr., Apt. \# 57,

Ann Arbor, MI 48105, U.S.A.

(Received: June 29, 1995) 